\title{
Feasibility of Capacitive Sensing of Surface Electromyographic Potential through Cloth
}

\author{
Akinori Ueno*, Toshihiko Yamaguchi ${ }^{1}$, Toshiya Iida ${ }^{1}$, \\ Yutaka Fukuoka $^{2, * *}$, Yoshinori Uchikawa ${ }^{3}$ and Makoto Noshiro ${ }^{4}$ \\ Department of Electrical and Electronic Engineering, Tokyo Denki University, \\ Kanda-Nishiki-cho, Chiyoda-ku, Tokyo 101-8457, Japan \\ 'Department of Electronic and Computer Engineering, Tokyo Denki University, \\ Ishizaka, Hatoyama-machi, Saitama 350-0394, Japan \\ ${ }^{2}$ Graduate School of Biomedical Science, Tokyo Medical and Dental University, \\ Yushima, Bunkyo-ku, Tokyo 113-8510, Japan \\ ${ }^{3}$ Division of Electrical and Mechanical Engineering, Tokyo Denki University, \\ Ishizaka, Hatoyama-machi, Saitama 350-0394, Japan \\ ${ }^{4}$ Department of Clinical Engineering, School of Allied Health Sciences, Kitasato University, \\ Kitasato, Sagamihara-shi, Kanagawa 228-8555, Japan
}

(Received July 21, 2011; accepted January 16, 2012)

Key words: capacitive electrode, EMG measurement, guarding technique, drive-ground-plane technique, human-machine interfaces

A capacitive sensing method was developed for sensing surface electromyographic potential (sEMG) through a thin cloth. The method is based on capacitive coupling involving fabric electrodes, the cloth, and the skin of the subject. A proposed measuring device was assembled by modifying the previously developed instrument for electrocardiographic potential. Experimental results showed that sEMG obtained with the proposed system was clearly visible and showed firings synchronized with sEMG simultaneously measured with a commercial device and that the spectral powers of both sEMGs were almost identical. These results demonstrated the potential of the proposed method to measure sEMG through cloth. The method enables the discomfort due to conventional skin-to-electrode coupling to be eliminated, although the signal quality, such as the signal-to-noise ratio $(\mathrm{S} / \mathrm{N})$, is slightly decreased with the cloth barrier.

\section{Introduction}

Since the late 1970s, myoelectric potential (or electromyogram: EMG) has been studied and used for biosignal-based mechatronics interfaces such as artificial limb

*Corresponding author: e-mail: ueno@mail.dendai.ac.jp

${ }^{* *}$ Present affiliation: Department of Electrical Engineering, Kogakuin University, Tokyo, Japan 
prostheses, ${ }^{(1-4)}$ robot hands, ${ }^{(5)}$ and manipulators. ${ }^{(6)}$ Recently, the EMG has opened up new possibilities for other human-computer interfaces in the fields of augmentative and alternative communication ${ }^{(7-10)}$ and environmental control..$^{(11)}$ The EMG contains information on muscle recruitment, timing pattern, and force. Therefore, the EMG is useful as an input signal for human adaptive mechatronics. ${ }^{(12)}$

In the conventional surface EMG (sEMG) measurement, an electrolytic paste or a conductive adhesive is almost always required for maintaining reliable ohmic contact with the skin. Therefore, a long-term sEMG measurement by the conventional method causes irritation and discomfort and is a potential cause of skin allergy and inflammation. These are considerably disadvantageous for the use of sEMG in human-machine interfaces because practical interfaces must be as noninvasive and nonintrusive as possible to gain broad acceptance from ordinary users.

To overcome the above disadvantages, we have focused on capacitive sensing, which allows the detection of alternating electrical potential through an inserted thin insulator. Capacitive sensing has been widely applied to measuring electrocardiograms (ECGs). ${ }^{(13-19)}$ Recent progresses were surveyed in a methodological review by Chi et al. ${ }^{(20)}$ A capacitive sensing system developed for ECG, however, cannot be simply applied to sEMG because of the following reasons. First, the frequency ranges are different between ECG and EMG. Second, the electrode should be smaller in EMG sensing than in ECG sensing because a large electrode would easily suffer from interference from adjacent muscles. In this study, a new capacitive sensing system is developed for sEMG, which enables the measurement of sEMG through commonly available cloth, and its feasibility is explored.

\section{Capacitive Sensing}

Capacitive sensing is based on the principle of the capacitive (or insulated) electrode, which is an electrode requiring no electrolytic paste or conductive adhesive. Conductive electrodes are capacitively coupled with the skin through an insulator (Fig. 1). The electrodes can carry an alternating bioelectrical current equivalently through the capacitance of each coupling. In previous studies, various insulators, such as anodized aluminum, ${ }^{(21,22)}$ silicon dioxide, ${ }^{(23,24)}$ pyre varnish, ${ }^{(25)}$ anodic insulated tantalum oxide, ${ }^{(26)}$ and barium titanate(27) were tested because these materials exhibit high permittivity. Capacitive sensing is facilitated with an impedance-transforming circuit to match the high impedance of the coupling to the low impedance required by the subsequent circuitry. A field-effect transistor (FET) source follower has been used as an impedancetransforming circuit, and is sometimes mounted in an electrode housing to reduce the common mode interference caused by power lines. ${ }^{(23,25)}$

\section{Materials and Methods}

\subsection{Flexible fabric electrode}

The sEMG signal was picked up using three electrodes, each of which was made of a rectangular sheet $\left(20 \times 50 \mathrm{~mm}^{2}\right)$ of conductive fabric with conductive acrylic adhesive (Kitagawa Industries, CSTK-100). Commonly available cloth, such as woven cotton, 


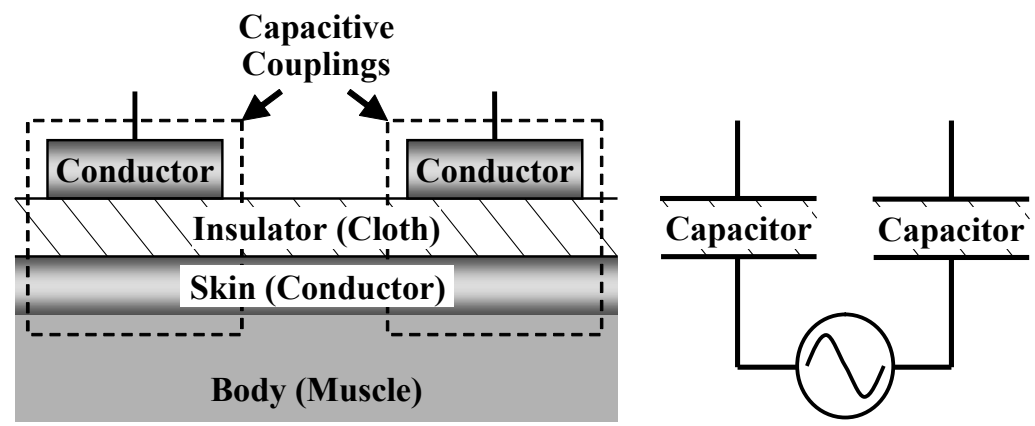

Fig. 1. Schematic model of two capacitive electrodes coupled to the skin with inserted cloth and its equivalent circuit.

was substituted for the rigid insulators. This enabled the insulator and electrodes to deform and achieve a closer fit to the coupled region, and accordingly, no electronic circuit was mounted in the electrode housing. The cloth insulator would also relieve the irritation, allergy and discomfort experienced with conventional skin-to-electrode coupling. The electrodes were attached to an insulating support made of flexible chloroethylene, and a convex connector (diameter: $5 \mathrm{~mm}$ ) was inserted between the support and each electrode (Fig. 2). The two lead electrodes at both ends were connected to a measuring device, to be described in the next section, with shielded wires. The center electrode was used as a reference electrode to reduce common mode noise as much as possible. The skin-cloth electrode coupling was held together by a rubber band.

\subsection{Amplifier unit}

An amplifier unit with filtering and amplification circuitry was assembled by modifying the measuring circuit developed for the capacitive sensing of ECG. ${ }^{(13)}$ In what follows, we will refer to this unit as a measuring device. The sensing circuitry for sEMG is more sensitive to power line interference because of the differences in the frequency ranges and the size of the electrodes between ECG and EMG sensing. Two major modifications were made to minimize the interference. First, the guarding technique was employed in the lead wires $(550 \mathrm{~mm})$ between an instrumentation amplifier and the electrodes to reduce the influence of leakage current. Second, the driven-ground-plane (DGP) technique( ${ }^{(28)}$ was introduced to improve the signal-to-noise ratio $(\mathrm{S} / \mathrm{N})$. This technique is a modification of the driven-rightleg (DRL) technique, ${ }^{(29)}$ which has been widely used in ECG measurement. In the DRL technique, the residual of common mode noise between the two differential inputs is inverted and directly fed back to the body earth electrode. Several groups have demonstrated its effect in reducing common mode noise. ${ }^{(15,19,30,31)}$ Similarly, in the DGP technique, the feedback is accomplished via an electrically noncontact reference electrode. The center electrode in Fig. 2 was used for this feedback. 


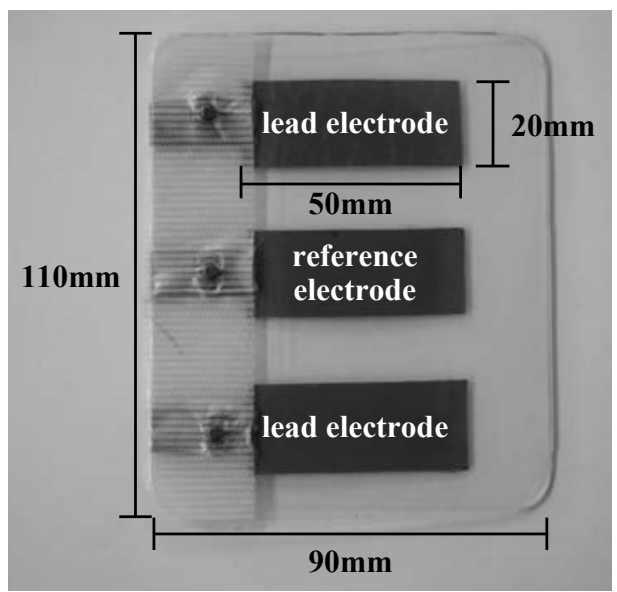

Fig. 2. Image of the measuring electrode unit made of conductive fabric and mounted on a flexible insulating support.

Figure 3 shows the electronic circuitry and element values of the measuring device. The device consists of two voltage followers, an instrumentation amplifier, a high-pass filter, a notch filter (NF Corporation, SR-2BE), a low-pass filter and three inverting amplifiers. As shown in Fig. 3, the voltage followers are incorporated at the front-end in the instrumentation amplifier IC. These voltage followers function as the impedancetransforming circuit to match the large coupling impedance that is due to the small dielectric constant of the inserted cloth. The actual input impedance of the IC on a substrate was $1.0 \mathrm{G} \Omega$ at $1.0 \mathrm{~Hz}$. The circuit constants in the high- and low-pass filters were set to obtain a cutoff frequency of 20 and $1,000 \mathrm{~Hz}$, respectively. The notch filter was used to reduce the $50 \mathrm{~Hz}$ power line interference. The impedance-transforming circuit was not mounted in the electrode housing so as to avoid a hard contact with the skin.

\subsection{Measurement of frequency characteristics of the measuring device}

The frequency characteristics of the developed measuring device were investigated using synthetic skin. The experimental setup is shown in Fig. 4. Measuring electrodes having identical areas of $1,000 \mathrm{~mm}^{2}$ were placed on an insulating support and covered with a cotton sheet $350 \mu \mathrm{m}$ thick. Then, three sheets of synthetic skin made of square pieces of conductive fabric having the same area as the measuring electrode were attached to the cloth at locations just above each of the measuring electrodes, so that the whole area of each measuring electrode was involved in capacitive coupling with each sheet of synthetic skin. Using a weight, a pressure of $424 \mathrm{~Pa}$ was applied to the electrodes, the cloth and the synthetic skin. This condition was selected so that it was slightly lower than the mean pressure exerted by the rubber band. A sinusoidal wave 


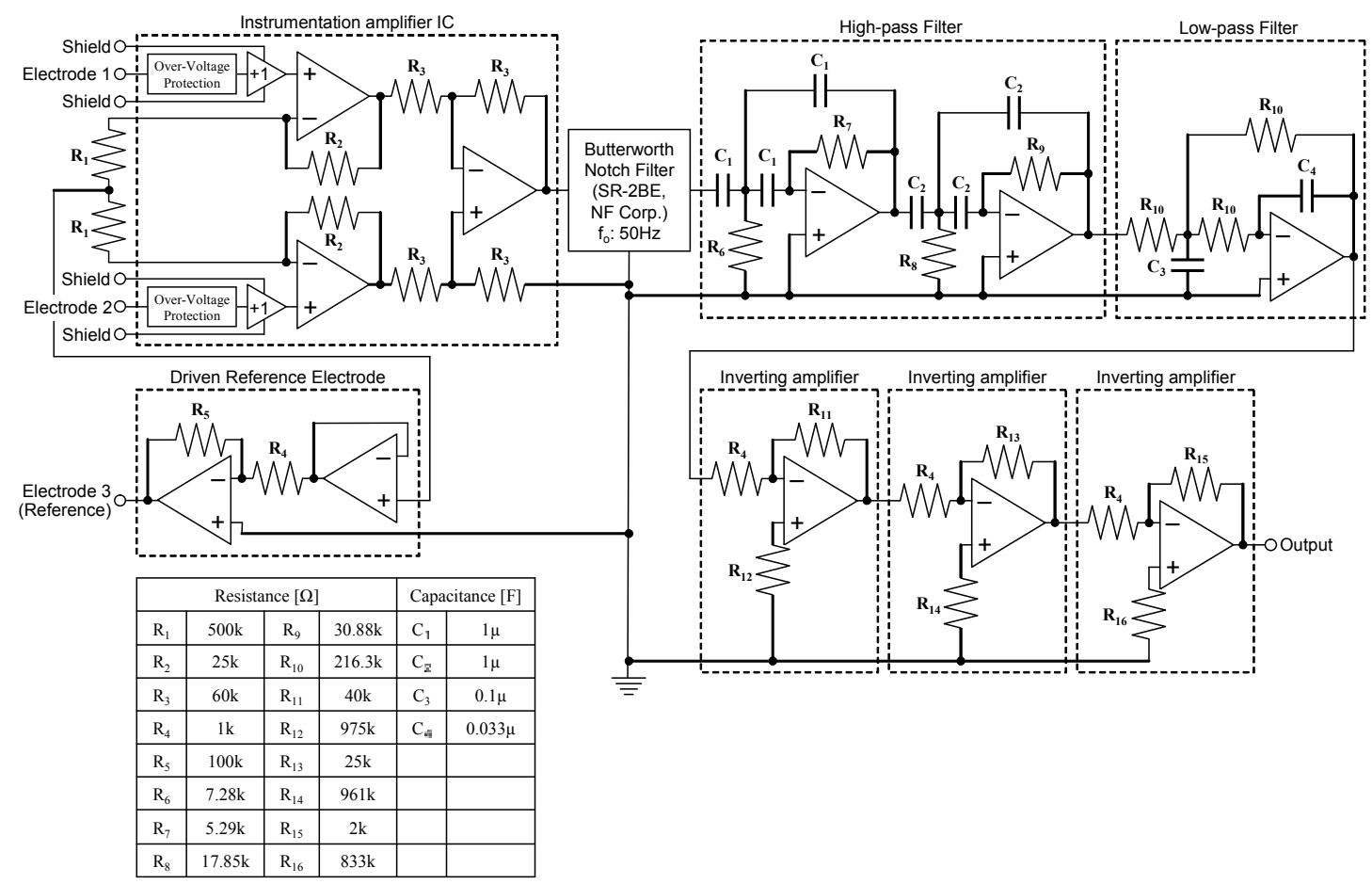

Fig. 3. Electronic circuitry and element values of the measuring device.

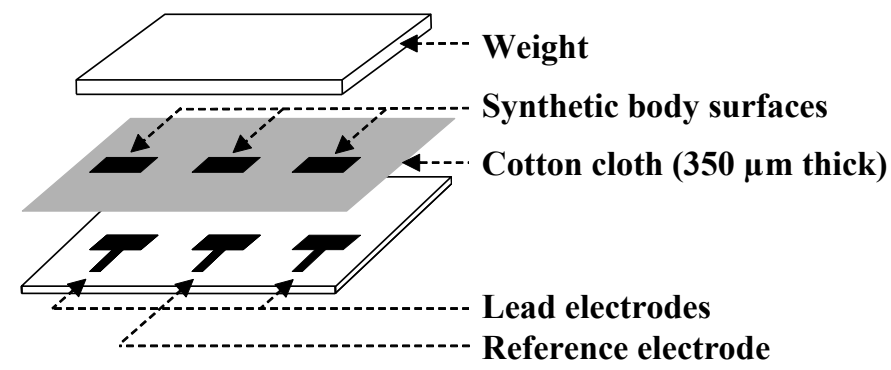

Fig. 4. Experimental setup for measuring the frequency response of the developed device.

was input from an oscillator to the synthetic skin and the output from the device was measured. This combination of inputting and measurement was conducted repeatedly within a frequency range from 1 to $5,000 \mathrm{~Hz}$. A frequency response without any cloth 
inserted was also measured for the same frequency range. In addition, $\mathrm{S} / \mathrm{N}$ and $\mathrm{CMRR}$ with and without the cloth were measured at $100 \mathrm{~Hz}$ using the same setup. The sEMG obtained without the cloth corresponded to the conventional pasteless, direct-contact measurement. The room temperature was $22^{\circ} \mathrm{C}$ and the relative humidity was $38 \%$ during the measurements.

\subsection{Comparison to a commercial device}

To compare sEMG signals obtained by the proposed and conventional methods, a simultaneous measurement was performed. One male volunteer, aged 21, participated in the measurement. The surface of the right brachium of the subject was covered with a cotton cloth $350 \mu \mathrm{m}$ thick. The unit of flexible electrodes was clamped on the right biceps brachii muscle over the cloth. An sEMG signal was recorded using the developed device from the site while the subject repeated isometrical contraction and relaxation of the muscles. A directly measured sEMG signal was recorded using a commercial bioamplifier (Teac Instruments, BA1104M) and a commercial telemeter unit (Teac Instruments, TU-4). Two disposable electrodes (Nihon Kohden, Vitrode F-150M) were attached directly to the skin at both sides of the flexible electrode unit along the muscle. A body earth electrode for conventional measurement was placed at the left elbow. The sEMG signals obtained with the developed and commercial devices were simultaneously digitized at intervals of $100 \mu$ s with 16-bit resolution using an A/D converter and stored in a personal computer using a data acquisition system (Biopac Systems, MP-150 system). The bandwidth of the commercial device was 5.3-1,000 Hz. Another measurement was conducted for the rectus femoris muscle of the left thigh in a similar way. The room temperature was $23^{\circ} \mathrm{C}$ and the relative humidity was $24 \%$ during the measurements.

\subsection{Comparison of mean power spectra}

The volunteer for the simultaneous measurement also participated in another experiment to measure the power spectra. First, an sEMG signal from the left rectus femoris muscle was measured through the $350-\mu \mathrm{m}$-thick cloth using the developed device while the subject repeated contraction and relaxation ten times. Then, an sEMG signal from the same site, but without any cloth, was measured using the same device during ten repetitive contractions. After the measurements, 10 segments $(800 \mathrm{~ms})$ of the sEMG signal were selected from each recording, and a power spectrum was calculated for each segment by FFT. Finally, the mean power spectrum was computed from the 10 spectra for each recording. The power spectra with and without the inserted cloth were compared. The latter corresponds to the conventional pasteless, direct-contact approach. The room temperature was $25^{\circ} \mathrm{C}$ and the relative humidity was $24 \%$ during the measurements.

\subsection{Influence of relative humidity on the signal}

The relative humidity of the room air is considered to influence the signal quality. Accordingly, this influence was investigated. The impedance of the inserted cotton was measured with a precision LCR meter (Agilent Technologies, 428A) at different relative 
humidities $(21,30,40$, and 51\%). Then, the measured data were fitted by

$$
\left|Z_{\text {cloth }}(f)\right|=\sqrt{\frac{\left(R_{\text {cont }}+R_{\text {cloth }}\right)^{2}+\left(2 \pi f C_{\text {cloth }} R_{\text {cont }} R_{\text {cloth }}\right)^{2}}{1+\left(2 \pi f C_{\text {cloth }} R_{\text {cloth }}\right)^{2}}}
$$

where $C_{\text {cloth }}$ and $R_{\text {cloth }}$ are the capacitance and DC resistance of the cloth, respectively, $R_{\text {cont }}$ is the contact resistance between the cloth and the conductive surfaces, and $f$ is the frequency. The equation was derived using the model of the cloth shown in Fig. 5.

\section{Results}

\subsection{Frequency characteristics of the measuring device}

The frequency-gain response without any inserted cloth (labeled "Conventional") showed a flat gain of about $66 \mathrm{~dB}$ within the frequency range between 20 and 1,000 $\mathrm{Hz}$, except at $50 \mathrm{~Hz}$, as designed (Fig. 6). The CMRR was $67 \mathrm{~dB}$ (Table 1). These characteristics meet the requirements of the Japanese Industrial Standard (JIS) for electromyography, and thus, the developed device can be used to measure an ordinary EMG when the electrode unit is attached directly to the skin. With the $350-\mu \mathrm{m}$-thick cloth inserted (labeled "Proposed" in Fig. 6), the frequency-gain response had almost a flat gain of around $56 \mathrm{~dB}$ within the frequency range between 20 and 1,000 Hz.

\subsection{Comparison to commercial device}

Figure 7 shows typical recordings obtained from the biceps brachii muscle and the rectus femoris muscle. The output signal of the developed device showed firings synchronized with the sEMG obtained with the commercial device. The waveforms, however, were not completely identical because of a slight difference in the detection site. The greater noise was probably due to the higher input impedance of the developed system.

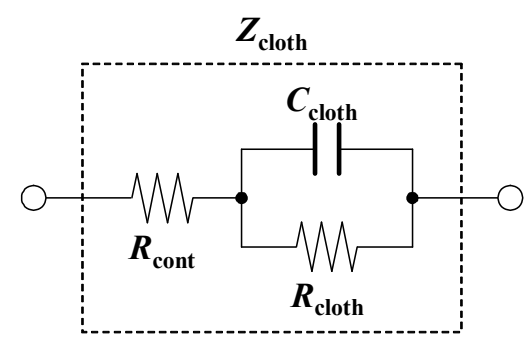

Fig. 5. Equivalent circuit of the cloth. $R_{\text {cont }}$ is the contact resistance between the cloth and conductive surfaces such as the electrode or skin. $R_{\text {cloth }}$ and $C_{\text {cloth }}$ are the DC resistance and capacitance of the cloth, respectively. 


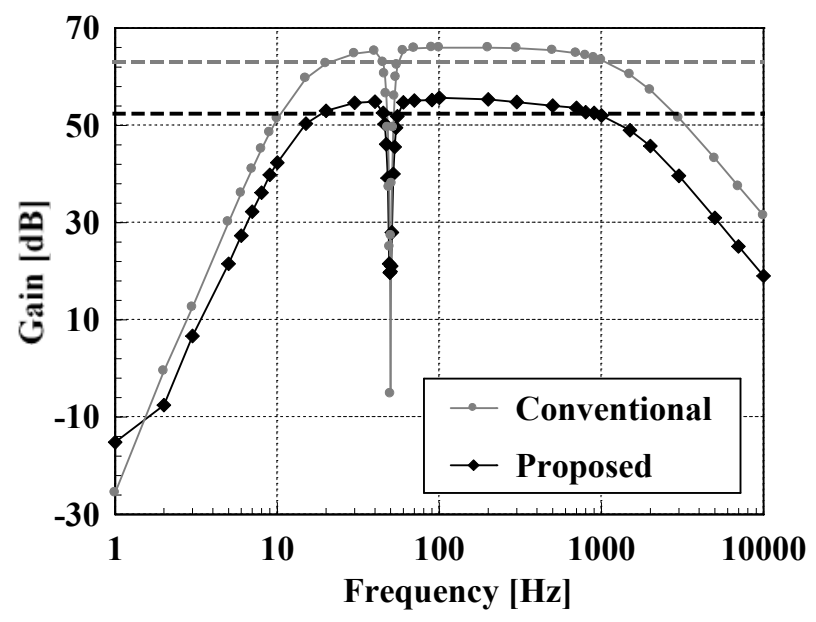

Fig. 6. Frequency-gain responses measured directly from synthetic skin (labeled "Conventional") and with the 350- $\mu \mathrm{m}$-thick cloth inserted (labeled "Proposed"). Grey and black dashed horizontal lines indicate $-3 \mathrm{~dB}$ from the maximum gain under each condition. The gain at $50 \mathrm{~Hz}$ was sharply attenuated because a notch filter was used in the device.

Table 1

Specifications of the developed device under conditions with and without the $350-\mu \mathrm{m}$-thick cotton cloth. The room temperature was $22^{\circ} \mathrm{C}$ and the relative humidity was $35 \%$ during the CMRR measurement.

\begin{tabular}{lcc}
\hline & Without cloth (direct) & With cotton cloth (424 Pa) \\
\hline Gain & $66 \mathrm{~dB}$ & $56 \mathrm{~dB}$ \\
Pass band & $20-1,000 \mathrm{~Hz}$ & $20-1,000 \mathrm{~Hz}$ \\
S/N at $100 \mathrm{~Hz}$ & $15.4 \mathrm{~dB}$ & $9.0 \mathrm{~dB}$ \\
CMRR at $100 \mathrm{~Hz}$ & $67 \mathrm{~dB}$ & $38 \mathrm{~dB}$ \\
\hline
\end{tabular}

\subsection{Comparison of mean power spectra}

Figure 8 shows the mean power spectra obtained with and without cloth insertion using the developed device. At frequencies between 20 and $500 \mathrm{~Hz}$, no notable differences were observed between the two power spectra, except for increases in power at harmonic frequencies of the $50 \mathrm{~Hz}$ power line, such as 250,350 , and $450 \mathrm{~Hz}$. Thus, alteration of the frequency components in the obtained sEMG resulting from cloth insertion seemed minimal in this frequency range. In contrast, different slopes were observed in a higher frequency band above $500 \mathrm{~Hz}$.

\subsection{Influence of relative humidity on the signal}

The frequency characteristics of the impedance of the inserted cloth are shown in Fig. 9. Black circles represent the measured impedance at the humidity of $21 \%$. Similarly, the 


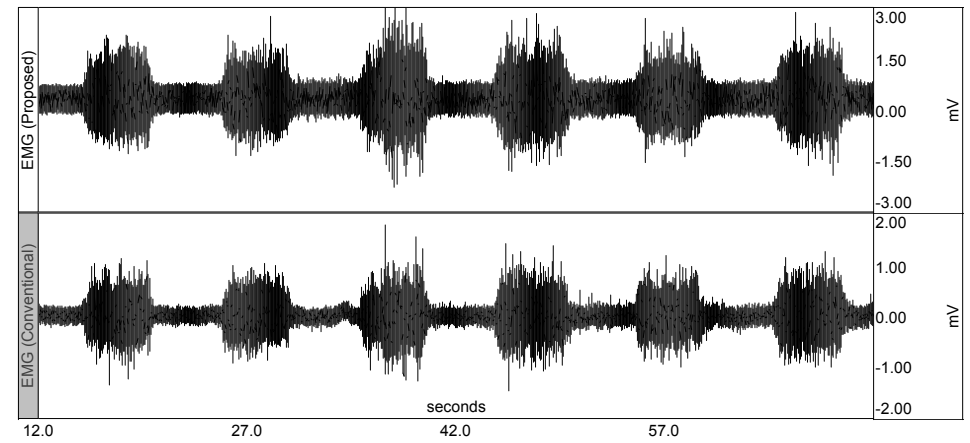

(a)

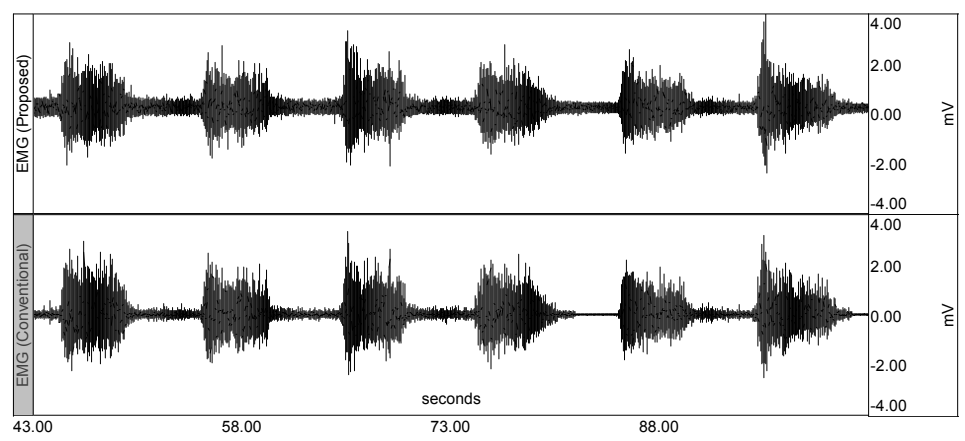

(b)

Fig. 7. Simultaneous recordings from (a) biceps brachii muscle and (b) rectus femoris muscle using the developed device with a cotton sheet $350 \mu \mathrm{m}$ thick (top) and using a commercial electromyograph without any cloth (bottom).

impedance was measured at humidity levels of 30\% (grey circles), $40 \%$ (black triangles), and $51 \%$ (grey triangles). The lines in the graph indicate the best-fit curves obtained by applying eq. (1) to the measured impedance data. The impedance of the cloth decreases as the relative humidity increases, particularly in a low-frequency range.

\section{Discussion}

\subsection{Feasibility of capacitive sensing of $S E M G$}

As shown in Fig. 6, the frequency responses with and without the cloth are similar, although the $\mathrm{S} / \mathrm{N}$ and CMRR were reduced by 6.4 and $29 \mathrm{~dB}$, respectively. The recordings obtained from the biceps brachii and rectus femoris muscles (Fig. 7) showed firings synchronized with those obtained with the commercial device. These results demonstrate the feasibility of the developed system for detecting sEMG even with the 


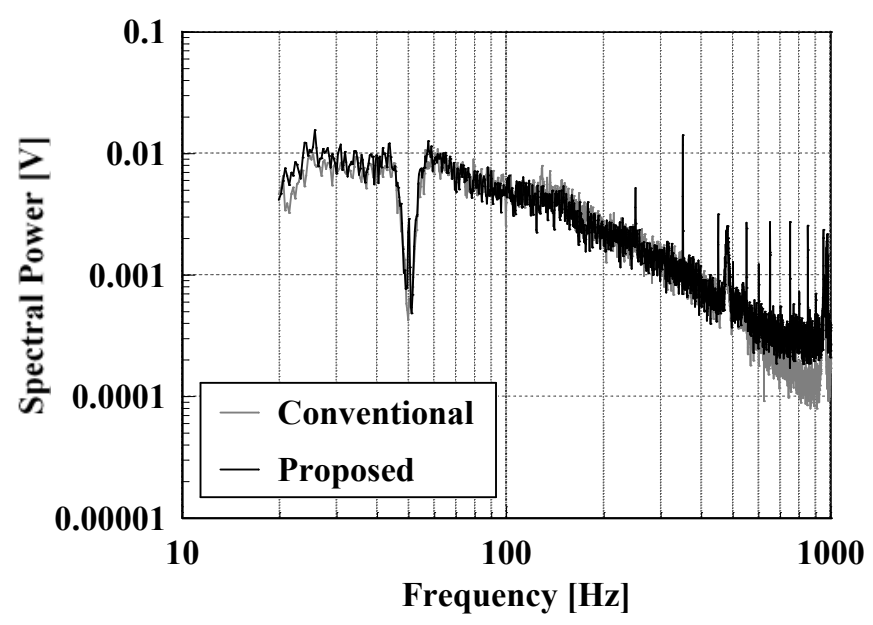

Fig. 8. Mean power spectra of sEMG measured from the rectus femoris muscle by the proposed and commercial methods.

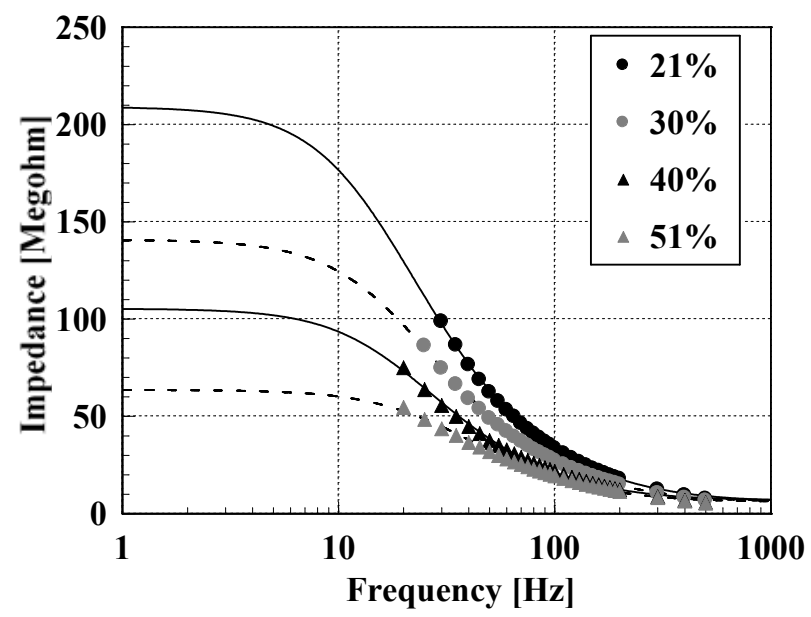

Fig. 9. Frequency-impedance responses of the inserted cloth in room air at different humidities. The data were measured with a precision LCR meter (Agilent Technologies, 4284A). Solid lines in the graph are the best-fit curves obtained by applying eq. (1).

cloth inserted, in particular, for nonclinical, human-machine interface applications. It should be noted that without the two modifications from the system for ECG, negligible sEMG signals were obtained with the cloth inserted (data not shown). 
There are some issues to be addressed in the future. First, the $\mathrm{S} / \mathrm{N}$ and CMRR remain to be further improved. Another important issue is the suppression of the harmonics of the $50 \mathrm{~Hz}$ power line in the signal, because the increase in the spectral power in the frequency range above $500 \mathrm{~Hz}$ with the cloth insertion may be caused by the accumulation of harmonics power.

\subsection{Influence of relative humidity on the signal}

As shown in Fig. 9, the relative humidity of the room air influences the impedance of the inserted cloth, and thus, the quality of the resulting sEMG signal. The relative humidity during the simultaneous measurements and the power spectra measurements was $24 \%$, which was lower than the relative humidity observed under ordinary circumstances. The $\mathrm{S} / \mathrm{N}$ of the output signal from the developed system can be improved if the measurement is conducted in a more ordinary, humid environment.

\subsection{Influence of perspiration on the signal}

Geddes and Valentinuzzi ${ }^{(32)}$ reported that dry electrodes were really dry only when first applied because of subsequent perspiration, and that contact impedance 20 min after electrode application became between one-fourth and one-fifth the initial impedance. During capacitive sensing with a cloth, a similar phenomenon will presumably occur, particularly in a long-term measurement. Because the decrease in the impedance of the cloth reduces the voltage loss at the electrode-cloth-skin coupling, perspiration will increase the amplitude of the output signal and bring it close to the level measured using directly attached electrodes. Furthermore, the decline in the impedance of the coupling is thought to reduce the interference caused by power lines. Perspiration will thus improve the $\mathrm{S} / \mathrm{N}$ of the output signal. In fact, we have observed an improvement in $\mathrm{S} / \mathrm{N}$ when the ECG was capacitively measured during sleep through cloth on the dorsal surface of the subjects. ${ }^{(13)}$

\section{Conclusions}

We have proposed a new approach for obtaining the sEMG under conditions whereby a thin cloth is inserted between the electrodes and the skin. We fabricated a prototype measuring device incorporating this approach, and validated the proposed approach. The examination of the developed system yielded the following results.

1. The frequency-gain responses indicated that the measuring system was capable of detecting sEMG not only when the electrodes were attached directly to the skin, but also when a $350-\mu \mathrm{m}$-thick cotton cloth was inserted between the electrodes and the skin.

2. The proposed method was able to yield a clearly visible sEMG from the biceps brachii muscle and rectus femoris muscle even with the cloth inserted, despite the fact that $\mathrm{S} / \mathrm{N}$ and CMRR deteriorated to 6.4 and $29 \mathrm{~dB}$, respectively, upon cloth insertion. Because the method eliminates discomfort due to conventional skin-to-electrode coupling, it appears promising for application to wearable sEMG sensors for humanmachine interfaces, such as Human Adaptive Mechatronics. 


\section{References}

1 D. Graupe, J. Magnussen and A. A. Beex: IEEE Trans. Autom. Control AC-23 (1978) 538.

2 S. C. Jacobson, D. F. Knutti, R. T. Johnson and H. H. Sears: IEEE Trans. Biomed. Eng. BME-29 (1982) 249.

3 E. Park and S. G. Meek: IEEE Trans. Biomed. Eng. 42 (1995) 1048.

4 D. Peleg, E. Braiman, E. Yom-Tov and G. F. Inbar: IEEE Trans. Neural Syst. Rehabil. Eng. 10 (2002) 290.

5 K. A. Farry, I. D. Walker and R. G. Baraniuk: IEEE Trans. Rob. Autom. 12 (1996) 775.

6 O. Fukuda, T. Tsuji, M. Kaneko and A. Otsuka: IEEE Trans. Rob. Autom. 19 (2003) 210.

7 A. B. Barreto, S. D. Scargle and M. Adjouadi: J. Rehabil. Res. Dev. 37 (2000) 53.

8 E. A. Goldstein, J. T. Heaton, J. B. Kobler, G. B. Stanley and R. E. Hillman: IEEE Trans. Biomed. Eng. 51 (2004) 325.

9 K. Miyazawa, A. Ueno, H. Mori, H. Hoshino and M. Noshiro: Conf. Proc. IEEE Eng. Med. Biol. Soc. (IEEE Service Center, Piscataway, 2006) p. 2518.

10 V. Stanford: IEEE Pervasive Comput. Mag. 3 (2004) 99.

11 H. Ogino, J. Arita and T. Tsuji: J. Rob. Mechatron. 17 (2005) 173.

12 K. J. Åstrom, M. Iwase, K. Furuta K and J. Åkesson: Int. J. Assist. Rob. Mechatron. 7 (2006) 3.

13 A. Ueno, Y. Akabane, T. Kato, H. Hoshino, S. Kataoka and Y. Ishiyama: IEEE Trans. Biomed. Eng. 54 (2007) 759.

14 A. Aleksandrowicz, M. Walter and S. Leonhardt: Biomed. Technik 52 (2007) 185.

15 Y. Yama, A. Ueno and Y. Uchikawa: Conf. Proc. IEEE Eng. Med. Biol. Soc. (IEEE Service Center, Piscataway, 2007) p. 5727.

16 M. Oehler, V. Ling, K. Melhorn and M. Schilling: Physiol. Meas. 29 (2008) 783.

17 T. Matsuda and M. Makikawa: Conf. Proc. IEEE Eng. Med. Biol. Soc. (IEEE Service Center, Piscataway, 2008) p. 1315.

18 M. Oehler, M. Schilling and H. D. Esperer: Biomed. Technik/Biomed. Eng. 54 (2009) 329.

19 A. Ueno, T. Imai, D. Kowada and Y. Yama: Biomedical Engineering, ed. C. A. Barros de Mello (INTECH, Vukovar, Croatia, 2009) Chap. 21, p. 399.

20 Y. M. Chi, T. P. Jung and G. Cauwenberghs: IEEE Rev. Biomed. Eng. 3 (2010) 106.

21 J. A. Lopez and P. C. Richardson: IEEE Trans. Biomed. Eng. BME-16 (1969) 99.

22 P. C. Richardson, F. K. Coombs and R. M. Adams: Aerosp. Med. 39 (1968) 7450.

23 W. H. Ko, M. R. Neuman, R. F. Wolfson and E. T. Yon: IEEE Trans. Industrial Electronics and Control Instrumentation IECI-17 (1970) p. 195.

24 R. N. Wolfson and M. R. Neuman: Proc. 8th Int. Conf. Med. Biol. Eng. (World Academy of Science, Engineering and Technology, Padova, 1969) Session 146.

25 A. Potter and L. Menke: IEEE Trans. Biomed. Eng. BME-17 (1970) 350.

26 C. H. Lagow, K. J. Sladek and P. C. Richardson: IEEE Trans. Biomed. Eng. BME-18 (1971) 162.

27 T. Matsuo, M. Esashi and K. Iinuma: Jpn. J. Med. Electron. Biol. Eng. 11 (1973) 10 (in Japanese).

28 K. K. Kim, Y. K. Lim and K. S. Park: Conf. Proc. IEEE Eng. Med. Biol. Soc. (IEEE Service Center, Piscataway, 2005) p. 5881.

29 E. M. Spinelli, N. H. Martinez and M. A. Mayosky: IEEE Trans. Biomed. Eng. 46 (1999) 1466.

30 K. F. Wu and Y. T. Zhang: Proc. 5th Int. Conf. Inf. Technol. Appl. Biomed. in conjunction with 2nd Int. Symp. Summer School Biomed. Health Eng. (IEEE Service Center, Piscataway, 2008) p. 282.

31 M. Steffen, A. Aleksandrowicz and S. Leonhardt: IEEE Trans. Biomed. Circuits Syst. 1 (2007) 250 .

32 L. A. Geddes and M. E. Valentinuzzi: Ann. Biomed. Eng. 1 (1973) 356. 